\title{
STAR FORMATION IN THE MOLECULAR CLOUD ASSOCIATED WITH THE MONKEY HEAD NEBULA: SEQUENTIAL OR SPONTANEOUS?
}

\author{
James O. Chibueze ${ }^{1,2}$, Kenji Imura ${ }^{1}$, Toshihiro Omodaka ${ }^{1}$, Toshihiro Handa $^{1}$, Takumi Nagayama ${ }^{3}$, Kenta Fujisawa ${ }^{4}$, \\ Kazuyoshi Sunada ${ }^{3}$, Makoto NaKano ${ }^{5}$, Tatsuya Kamezaki ${ }^{1}$, Yoshiyuki Yamaguchi $^{1}$, and Mamoru SeKido $^{6}$ \\ ${ }^{1}$ Department of Physics and Astronomy, Graduate School of Science and Engineering, Kagoshima University, \\ 1-21-35 Korimoto, Kagoshima 890-0065, Japan; james@milkyway.sci.kagoshima-u.ac.jp \\ ${ }^{2}$ Department of Physics and Astronomy, Faculty of Physical Sciences, University of Nigeria, Carver Building, 1 University Road, Nsukka, Nigeria \\ ${ }^{3}$ Mizusawa VLBI Observatory, National Astronomical Observatory of Japan, 2-21-1 Osawa, Mitaka, Tokyo 181-8588, Japan \\ ${ }^{4}$ Department of Physics and Informatics, Faculty of Science, Yamaguchi University, Yoshida 1677-1, Yamaguchi 753-8512, Japan \\ ${ }^{5}$ Faculty of Education and Welfare Science, Oita University, Oita 870-1192, Japan \\ ${ }^{6}$ Kashima Space Research Center, National Institute of Information and Communications Technology, 893-1 Hirai, Kashima, Ibaraki 314-8501, Japan \\ Received 2012 September 7; accepted 2012 October 27; published 2012 December 11
}

\begin{abstract}
We mapped the $(1,1),(2,2)$, and $(3,3)$ lines of $\mathrm{NH}_{3}$ toward the molecular cloud associated with the Monkey Head Nebula (MHN) with a 1'.6 angular resolution using a Kashima $34 \mathrm{~m}$ telescope operated by the National Institute of Information and Communications Technology (NICT). The kinetic temperature of the molecular gas is $15-30 \mathrm{~K}$ in the eastern part and $30-50 \mathrm{~K}$ in the western part. The warmer gas is confined to a small region close to the compact H II region S252A. The cooler gas is extended over the cloud even near the extended H II region, the MHN. We made radio continuum observations at $8.4 \mathrm{GHz}$ using the Yamaguchi $32 \mathrm{~m}$ radio telescope. The resultant map shows no significant extension from the $\mathrm{H} \alpha$ image. This means that the molecular cloud is less affected by the MHN, suggesting that the molecular cloud did not form by the expanding shock of the MHN. Although the spatial distribution of the Wide-field Infrared Survey Explorer and Two Micron All Sky Survey point sources suggests that triggered low- and intermediate-mass star formation took place locally around S252A, but the exciting star associated with it should be formed spontaneously in the molecular cloud.
\end{abstract}

Key words: H II regions - ISM: clouds - ISM: individual objects (Gemini OB1) - ISM: molecules

Online-only material: color figures

\section{INTRODUCTION}

The formation of a high-mass star leads to the ionization of its immediate surroundings and the formation of the $\mathrm{H}$ II region. There are many studies on the interactions between $\mathrm{H}$ II regions and nearby molecular clouds. One classical work suggested that a sequential star formation was triggered by the interstellar shock induced by the expanding H II region (Elmegreen \& Lada 1977). However, it is observationally difficult to derive the history of star formation in a single star-forming region. Applying an analytical and numerical method, Bonnell \& Bate (2006) argue that massive stars form through a competitive accretion process after a gravitational collapse. Assuming local properties rather than global variables, they showed that few stars located at the center of a local stellar system would initially accrete low relative velocity gas as a result of the influence of turbulence. Subsequently, after acquiring enough mass to maintain a high-accretion rate, the accretion of higher relative velocity gas begins and leads to the formation of high-mass stars. Most of the stars located away from the center of the stellar system, as well as those that enter the higher relative velocity gas region with insufficient mass to maintain a high-accretion rate, can only retain their low-mass status.

While there is some observational evidence of low- and intermediate-mass star formation triggered by the influence of an expanding $\mathrm{H}$ II region (Deharveng et al. 2009; Koenig et al. 2008), only a few massive stars are suggested to have formed through such a triggered process. Assuming that the Midcourse Space Experiment (MSX) identified associated embedded clusters as second generation clusters, Deharveng et al. (2005) suggested Sh 104, Sh 217, and RCW 79 to be triggering the formation of high-mass stars due to the presence of ultra-compact
H II regions. Thompson et al. (2012) suggested that only about $14 \%$ of the massive stars in the Milky Way could have been triggered, and in effect, the remaining $86 \%$ were formed through a different scenario. They also identified the phenomenological problem associated with triggered star formation studies, which is the preconceived expectation that the identified young stellar objects (YSOs) have formed through a triggered process.

S252 is an H II region catalogued by Sharpless (1959) and also known as the Monkey Head Nebula (MHN). It is located in an OB association, Gem OB1 (Morgan et al. 1953), and is suggested to be a prototypical site of sequential star formation (Efremov 1995). Its distance is estimated to be $2.1 \mathrm{kpc}$ using the annual parallax (Reid et al. 2009). There is clear evidence of star formation activities in this region. Carpenter et al. (1995b) found that 8 of 11 dense molecular gas clumps are associated IRAS point sources, and star clusters were found in some clumps through their near infrared survey.

The morphology of the molecular cloud suggests that there is interaction between the $\mathrm{H}$ II region and molecular gas. Carpenter et al. (1995a) made maps in ${ }^{12} \mathrm{CO}$ and ${ }^{13} \mathrm{CO}$ lines of the Gem OB1 region with the Five College Radio Astronomy Observatory $14 \mathrm{~m}$ telescope. Along the western edge of S252, a molecular cloud with a large column density is seen in their ${ }^{13} \mathrm{CO}$ map (see Figure 4). In addition, they found many arcs and ring-like filaments, which suggests that the molecular gas may have been swept up due to expanding H II regions and wind blown bubbles. Most of the arcs and filaments show high column density $\left(>10^{22} \mathrm{~cm}^{-2}\right)$ and a typical temperature range of $10-20 \mathrm{~K}$.

However, to study the interaction between the $\mathrm{H}$ II region and the molecular cloud, it is vital to investigate of the distribution of the dense gas and of the gas temperature. $\mathrm{NH}_{3}$ lines prove to be 
useful for investigating the physical conditions of a molecular cloud. Although Wu et al. (2010) made $\mathrm{NH}_{3}$ line observations toward this cloud with the Effersberg $100 \mathrm{~m}$ telescope, they only show the temperatures at three $\mathrm{NH}_{3}$ peaks. Therefore, we made the mapping observations in $\mathrm{NH}_{3}(1,1),(2,2)$, and $(3,3)$ lines and estimated the distribution of the gas kinetic temperature over the cloud. We also made observations in the radio continuum in order to explore the true distribution of ionized gas free from dust extinction.

\section{OBSERVATIONS}

\section{1. $\mathrm{NH}_{3}$ Observations}

In order to determine the distribution of the dense molecular gas and its temperature, we carried out the $\mathrm{NH}_{3}$ observations using the $34 \mathrm{~m}$ telescope at the Kashima Space Research Center at the National Institute for Communication Technology (NICT) between 2008 October and 2010 December. The simultaneous observations were done in the $\mathrm{NH}_{3}(J, K)=(1,1),(2,2)$, and $(3,3)$ lines, whose frequencies are $23.694495 \mathrm{GHz}, 23.722633 \mathrm{GHz}$, and $23.870129 \mathrm{GHz}$, respectively. The telescope beam size is 1'.6. We observed 160 positions with a $1^{\prime}$ grid along the equatorial coordinates over the cloud. The pointing accuracy was checked using several $\mathrm{H}_{2} \mathrm{O}$ maser sources at $22.235080 \mathrm{GHz}$ and was better than 0.2 . We used an 8192-channel FX-type spectrometer with a $256 \mathrm{MHz}$ bandwidth and $31.25 \mathrm{kHz}$ resolution, corresponding to $3200 \mathrm{~km} \mathrm{~s}^{-1}$ and $0.39 \mathrm{~km} \mathrm{~s}^{-1}$, respectively. All spectra were obtained with the position switch between the target source and a reference position. The reference position, where no ${ }^{13} \mathrm{CO}$ or $\mathrm{NH}_{3}$ is detected, is $(\alpha, \delta)_{\mathrm{J} 2000}=\left(06^{\mathrm{h}} 08^{\mathrm{m}} 26^{\mathrm{s}} .27\right.$, $\left.20^{\circ} 51^{\prime} 10^{\prime \prime} 7\right)$. The system noise temperature is between 170 and $350 \mathrm{~K}$. We integrated longer than 20 minutes at each position. The main beam efficiency, $\eta_{\mathrm{MB}}$, at this frequency is $0.50 \pm 0.02$. The resultant noise level in terms of the main beam temperature is about $0.1 \mathrm{~K}$ in $T_{\mathrm{MB}}=T_{A} / \eta_{\mathrm{MB}}$, where $T_{A}$ is the antenna temperature corrected for the atmospheric extinction using the chopper wheel method (Kutner \& Ulich 1981). We reduced the data using a software package named UltraSTAR developed by a radio astronomy group at the University of Tokyo (Nakajima et al. 2007). We applied a linear baseline to subtract all the spectra.

\subsection{Radio Continuum Observations}

The true distribution of ionized gases in $\mathrm{H}$ II regions can be obtained through radio continuum observations, which are not affected by dust extinction. Although the MHN is visible in optical images, it might be strongly obscured by local dense gas. In such cases, we cannot easily conclude that the dense gas directly interacts with the ionized gas. We obtained a $1.4 \mathrm{GHz}(20 \mathrm{~cm})$ radio continuum map of the region from the NRAO/VLA Sky Survey (NVSS) with a resolution of 45" (see http://www.cv.nrao.edu/nvss/postage.shtml). Because the NVSS map was obtained by a Very Large Array (VLA) interferometric observation, the likelihood of resolving the diffused component of the MHN is very high. We therefore made observations at $8.4 \mathrm{GHz}$ of the $\mathrm{MHN}$ with the $32 \mathrm{~m}$ telescope operated by Yamaguchi University. The telescope beam size is $4^{\prime}$, and its main beam efficiency at $8.4 \mathrm{GHz}$ is $0.70 \pm 0.04$. The system noise temperature was $\sim 70 \mathrm{~K}$, and the resultant noise level in terms of the main beam temperature $\left(T_{\mathrm{mb}}\right)$ is $0.04 \mathrm{~K}$.

The observation was performed on 2010 January 17, over an area of $2^{\circ} \times 2^{\circ}$ centering on the $\mathrm{H}$ II region. The raster-scan method was used: the telescope moved smoothly at a fixed
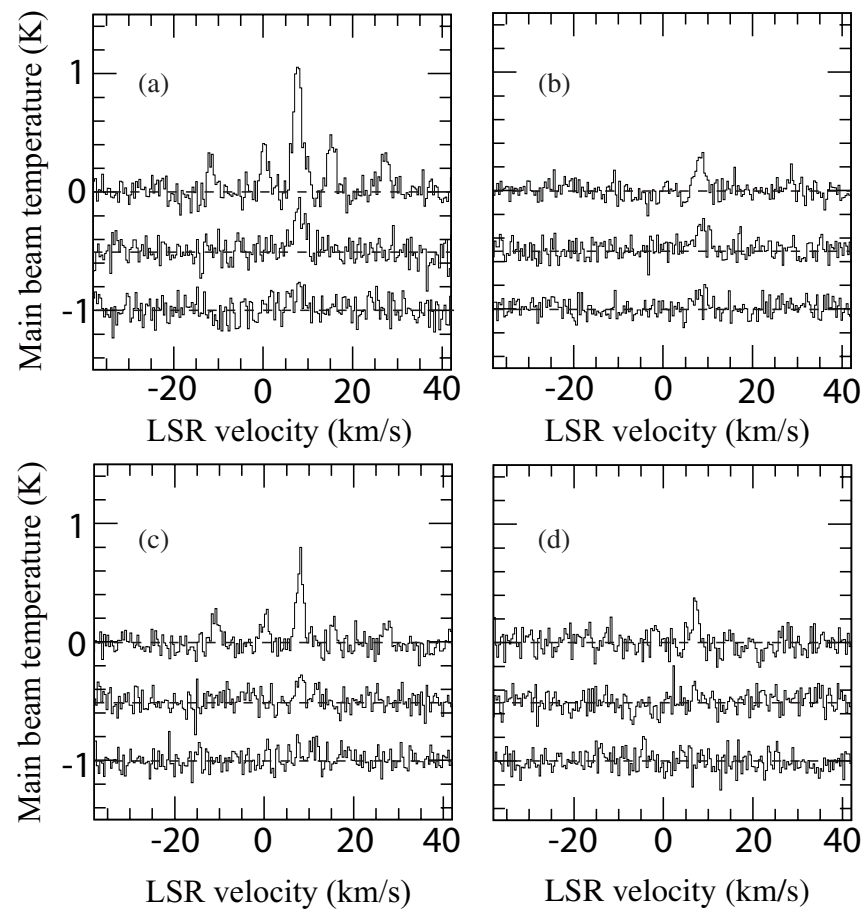

Figure 1. Typical spectra in the $\mathrm{NH}_{3}(J, K)=(1,1)$ (top spectra), $(2,2)$ (middle spectra), and $(3,3)$ (bottom spectra) lines. $(1,1)$ and $(2,2)$ transitions were detected at positions such as $(\mathrm{a}, \mathrm{b}$, and $\mathrm{c})$. Only the $(1,1)$ transition was detected at other positions. Two pairs of satellite lines of the $(1,1)$, the inner (satellite lines closest to and on both sides of the main line), and the outer (satellite lines farther away from and on both sides of the main line) were detected in some of the positions and weak in other positions.

speed in the declination direction, and the antenna temperature was measured continuously. The grid of the data was $1^{\prime}$ in both right ascension and declination. Since the FWHM of the beam is 4.2 , it is oversampled enough and the data can be smoothed during the data analysis. The observing bandwidth was $400 \mathrm{MHz}$ centered on $8380 \mathrm{MHz}$. Both the left-hand circular polarization (LHCP) and right-hand circular polarization (RHCP) were used simultaneously. For the calibration of the flux density and the observing position, the calibrator source $3 \mathrm{C} 138$, $(\alpha, \delta)_{\mathrm{J} 2000}=\left(05^{\mathrm{h}} 21^{\mathrm{m}} 09^{\mathrm{s}} .886,16^{\circ} 38^{\prime} 22^{\prime \prime} .051\right)$ of flux $2.44 \mathrm{Jy}$ at $8.4 \mathrm{GHz}$ was observed with the same observation system just before the observation of the $\mathrm{H}$ II region. The calibrator was observed for 36 minutes, followed by the observation of the $\mathrm{H}$ II region for $2 \mathrm{hr}$. Only the declination scan was performed due to the limited observing time. The system temperature was $50 \mathrm{~K}$ for LHCP and $45 \mathrm{~K}$ for RHCP. The observation weather was fine and the wind was weak. The obtained data were analyzed using a software developed locally. In order to reduce scanning noise, the PRESS method of Sofue \& Reich (1979) was used.

\section{RESULTS}

\subsection{Distribution of $\mathrm{NH}_{3} \mathrm{Gas}$}

We observed the $\mathrm{NH}_{3}$ lines at 160 positions in the mapping area. The $\mathrm{NH}_{3}(1,1)$ and $(2,2)$ were detected at 87 and 33 positions, respectively, with a signal-to-noise ratio $>3$. Figure 1 shows the typical profiles. Both the main and satellite lines of the $(1,1)$ transition were only detected at some positions, while the main lines of $(2,2)$ were detected most of the time. $\mathrm{NH}_{3}(3,3)$ was not detected at any position, and it will not be considered in this paper. 

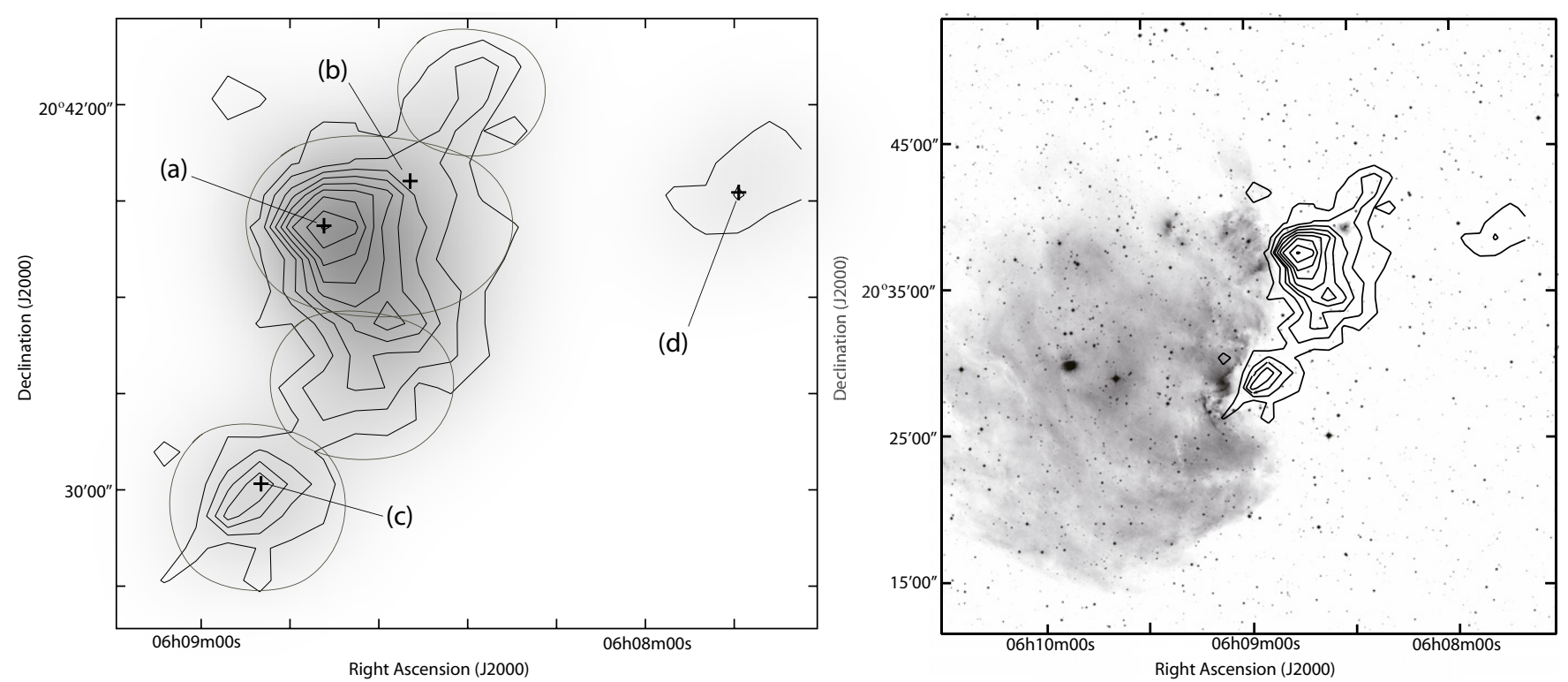

Figure 2. Left: integrated intensity map of the main hyperfine component of the $\mathrm{NH}_{3}(1,1)$ line. The lowest contour line and the contour interval are both $0.3 \mathrm{~K} \mathrm{~km} \mathrm{~s}{ }^{-1}$. (a)-(d) represent the positions where the spectra of Figure 1 were obtained. The inscribed gray-circled areas indicate the division of the molecular cloud into four parts for deriving its virial mass. Right: $\mathrm{NH}_{3}$ gas distribution along the edge of the ionized gas in the $\mathrm{H}$ II region of the MHN (H $\alpha$ image).

Table 1

Typical $\mathrm{NH}_{3}$ Spectra Parameters

\begin{tabular}{|c|c|c|c|c|c|c|}
\hline & R.A.(J2000) & Decl.(J2000) & $\begin{array}{l}\text { Rotational Transition } \\
\qquad(J, K)\end{array}$ & $\begin{array}{l}T_{\mathrm{MB}} \\
(\mathrm{K})\end{array}$ & $\begin{array}{c}v_{\mathrm{LSR}} \\
\left(\mathrm{km} \mathrm{s}^{-1}\right)\end{array}$ & $\begin{array}{c}\Delta v \\
\left(\mathrm{~km} \mathrm{~s}^{-1}\right)\end{array}$ \\
\hline $\mathrm{a}$ & $06^{\mathrm{h}} 08^{\mathrm{m}} 43^{\mathrm{s}} .48$ & $+20^{\circ} 38^{\prime} 10^{\prime \prime} 7$ & $\begin{array}{l}(1,1) \\
(2,2) \\
(3,3)\end{array}$ & $\begin{array}{l}1.0 \pm 0.1 \\
0.4 \pm 0.1 \\
0.2 \pm 0.1\end{array}$ & $\begin{array}{l}8.0 \pm 0.1 \\
8.3 \pm 0.2 \\
7.8 \pm 0.6\end{array}$ & $\begin{array}{l}2.7 \pm 0.2 \\
3.1 \pm 0.5 \\
4.9 \pm 2.0\end{array}$ \\
\hline $\mathrm{b}$ & $06^{\mathrm{h}} 08^{\mathrm{m}} 30.57$ & $+20^{\circ} 39^{\prime} 10^{\prime \prime} 7$ & $\begin{array}{l}(1,1) \\
(2,2) \\
(3,3)\end{array}$ & $\begin{array}{c}0.3 \pm 0.1 \\
0.2 \pm 0.1 \\
\ldots\end{array}$ & $\begin{array}{c}8.5 \pm 0.1 \\
9.0 \pm 0.5 \\
\ldots\end{array}$ & $\begin{array}{c}2.3 \pm 0.3 \\
4.3 \pm 1.4 \\
\ldots\end{array}$ \\
\hline $\mathrm{c}$ & $06^{\mathrm{h}} 08^{\mathrm{m}} 52^{\mathrm{s}} .09$ & $+20^{\circ} 30^{\prime} 10^{\prime \prime} 7$ & $\begin{array}{l}(1,1) \\
(2,2) \\
(3,3)\end{array}$ & $\begin{array}{c}0.7 \pm 0.1 \\
0.2 \pm 0.1 \\
\ldots\end{array}$ & $\begin{array}{c}8.2 \pm 0.1 \\
8.3 \pm 0.1 \\
\ldots\end{array}$ & $\begin{array}{c}1.7 \pm 0.2 \\
1.9 \pm 0.3 \\
\ldots\end{array}$ \\
\hline $\mathrm{d}$ & $06^{\mathrm{h}} 07^{\mathrm{m}} 47^{\mathrm{s}} .54$ & $+20^{\circ} 39^{\prime} 10^{\prime \prime} 7$ & $\begin{array}{l}(1,1) \\
(3,3) \\
(3,3)\end{array}$ & $\begin{array}{c}0.4 \pm 0.1 \\
\ldots \\
\ldots\end{array}$ & $\begin{array}{c}7.4 \pm 0.1 \\
\ldots \\
\ldots\end{array}$ & $\begin{array}{c}1.4 \pm 0.3 \\
\ldots \\
\ldots\end{array}$ \\
\hline
\end{tabular}

In Table 1, we show the Gaussian fitting values of the main beam brightness temperature $\mathrm{T}_{\mathrm{MB}}$, the peak velocity $v_{\mathrm{LSR}}$, and the FWHM $\Delta v$ at the four positions $(\mathrm{a}, \mathrm{b}$, c, and d) shown in Figure 2, whose spectra are shown in Figure 1. The radial velocities of the $\mathrm{NH}_{3}$ lines are between 7.4 and $8.9 \mathrm{~km} \mathrm{~s}^{-1}$, which is near that of the MHN, obtained from the $\mathrm{H}(167 \alpha)$ observations, where its velocity was measured as $4.9 \pm 2.2 \mathrm{~km} \mathrm{~s}^{-1}$ (Fountain et al. 1983).

Figure 2 shows the integrated intensity map of the main hyperfine component of the $\mathrm{NH}_{3}$ line over $v_{\mathrm{LSR}}=4-13 \mathrm{~km} \mathrm{~s}^{-1}$. It extends over $10 \mathrm{pc}$ around the northwest to southeast of the MHN (Figure 3). Figure 3 shows the integrated intensity map in the $\mathrm{NH}_{3}(1,1)$ line obtained at $\sigma>3$ superimposed on the ${ }^{13} \mathrm{CO}$ (green contour; Carpenter et al. 1995a) and CS maps (blue contour; Carpenter et al. 1995b), respectively. The $\mathrm{NH}_{3}$ emission traces the dense molecular gas well. Figure 4 shows the velocity channel maps between $v_{\mathrm{LSR}}=6-11 \mathrm{~km} \mathrm{~s}^{-1}$. It shows that the two core components are at $8 \mathrm{~km} \mathrm{~s}^{-1}$.

Our radio continuum map of the $\mathrm{H}$ II region at $8.4 \mathrm{GHz}$ shows similar morphology with the digital sky survey image (Kronberger et al. 2006). The white contour map overlaid on our $8.4 \mathrm{GHz}$ map (Figure 5) is the VLA map at $1.4 \mathrm{GHz}$ from the NVSS. Obviously, the VLA map misses the diffuse emission. The total extent of the radio continuum at $8.4 \mathrm{GHz}$ is similar to that in the optical image (see Figure 2). This means that the optical image is hardly obscured even at the edge of the dense molecular cloud seen in $\mathrm{NH}_{3}$ and traces the whole extent of the $\mathrm{H}$ II region well.

\subsection{Temperature of the Dense Molecular Cloud}

The gas kinetic temperature is important in discussing the physical state of a molecular cloud. The molecular cloud we observed is located at the edge of an extended $\mathrm{H}$ II region. Hence, the gas temperature should vary from place to place if the H II region affects the molecular cloud.

To estimate the gas kinetic temperature, we should know the optical depth for each line of sight, which can be derived from the line intensity ratios of the main and satellite lines. However, we can derive the optical depth only at some positions for the $(1,1)$ line, as shown in the previous subsection. Figure 6 shows the correlation between the $\mathrm{NH}_{3}(1,1)$ and the satellite lines, and its fittings show $\tau$ to typically be 0.9 . Therefore, we assume the optical depth is the same at all positions and $\tau=0.9$. 


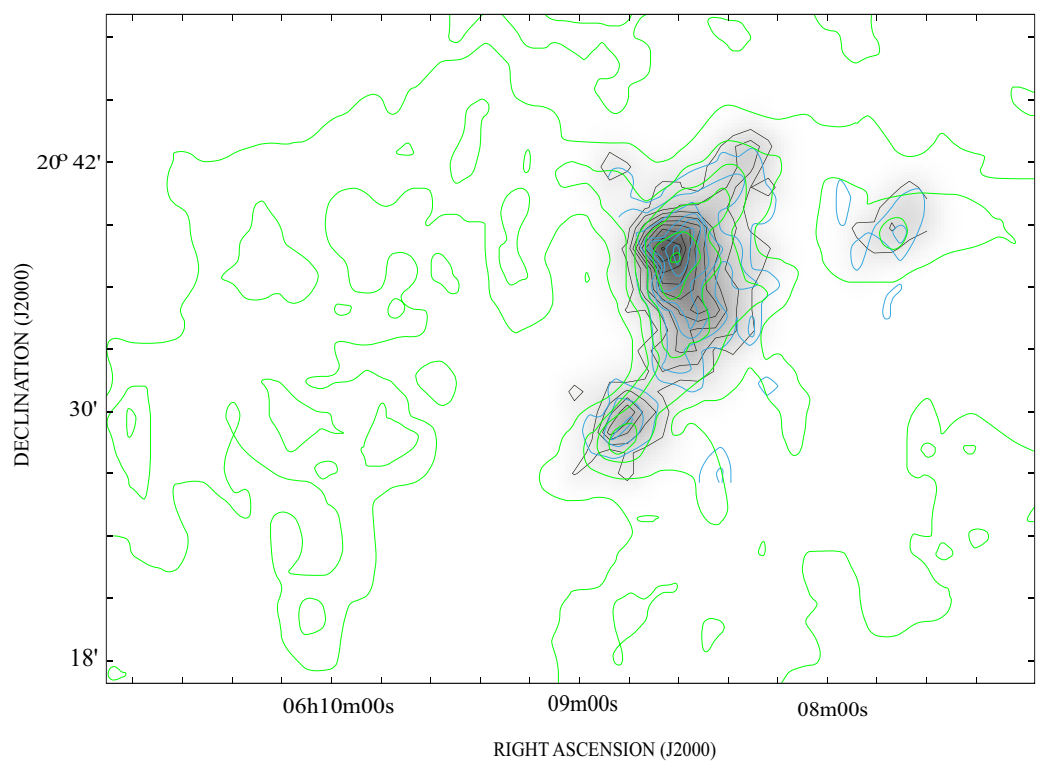

Figure 3. $\mathrm{NH}_{3}(1,1)$ integrated intensity map (shown in Figure 2) overlaid on the ${ }^{13} \mathrm{CO}(J=1-0)$ and CS map (Carpenter et al. 1995a). The black contours correspond to the $\mathrm{NH}_{3}(1,1)$ integrated intensity map, while the green and blue contours correspond to the ${ }^{13} \mathrm{CO}(J=1-0)$ and $\mathrm{CS}$ maps, respectively.

(A color version of this figure is available in the online journal.)

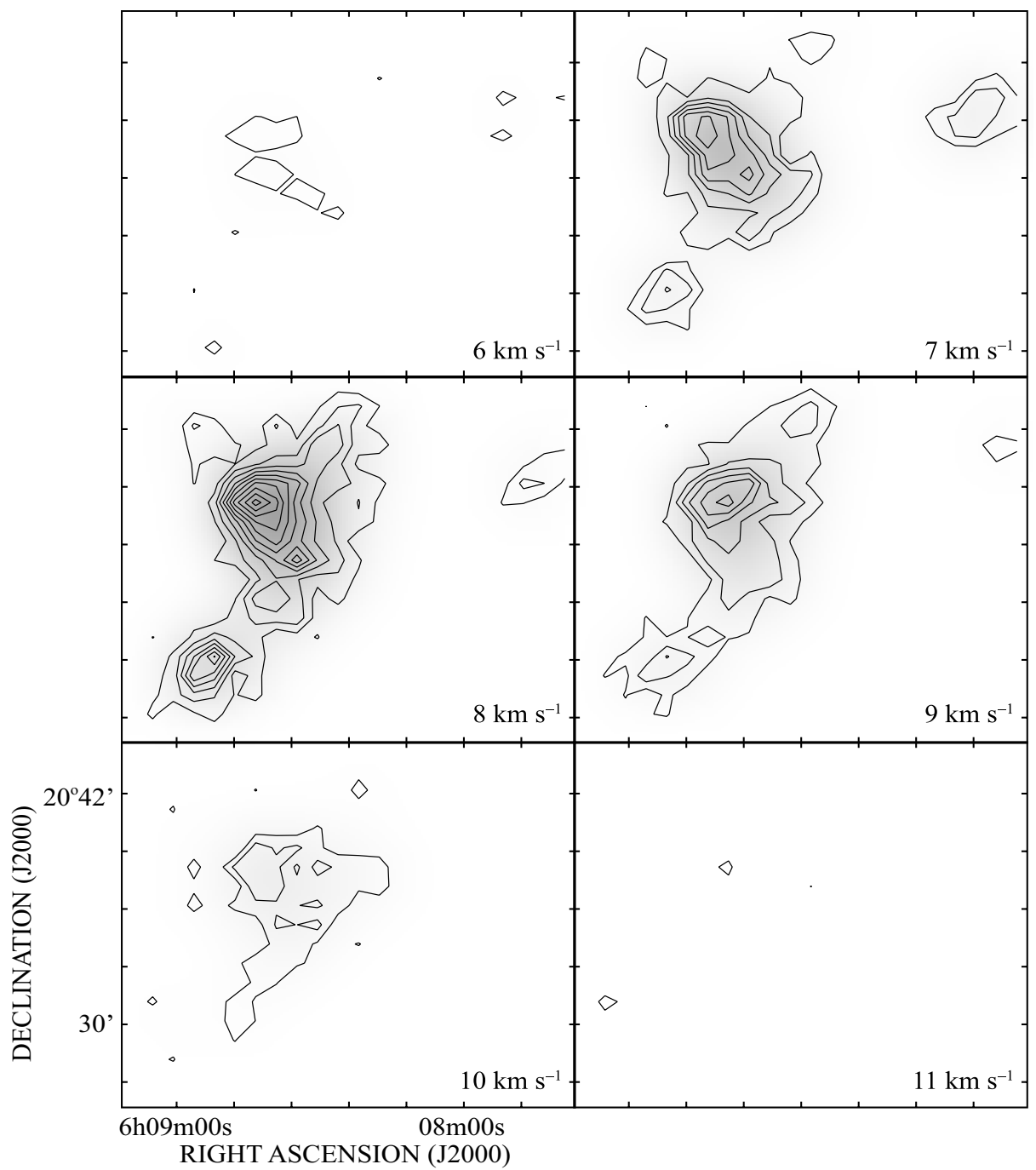

Figure 4. $\mathrm{NH}_{3}(1,1)$ channel map. The lowest contour line is $0.1 \mathrm{~K}$ and the contour interval is $0.1 \mathrm{~K}$ in $T_{\mathrm{MB}}$. The center velocity of each panel is shown at the bottom-right corner. 


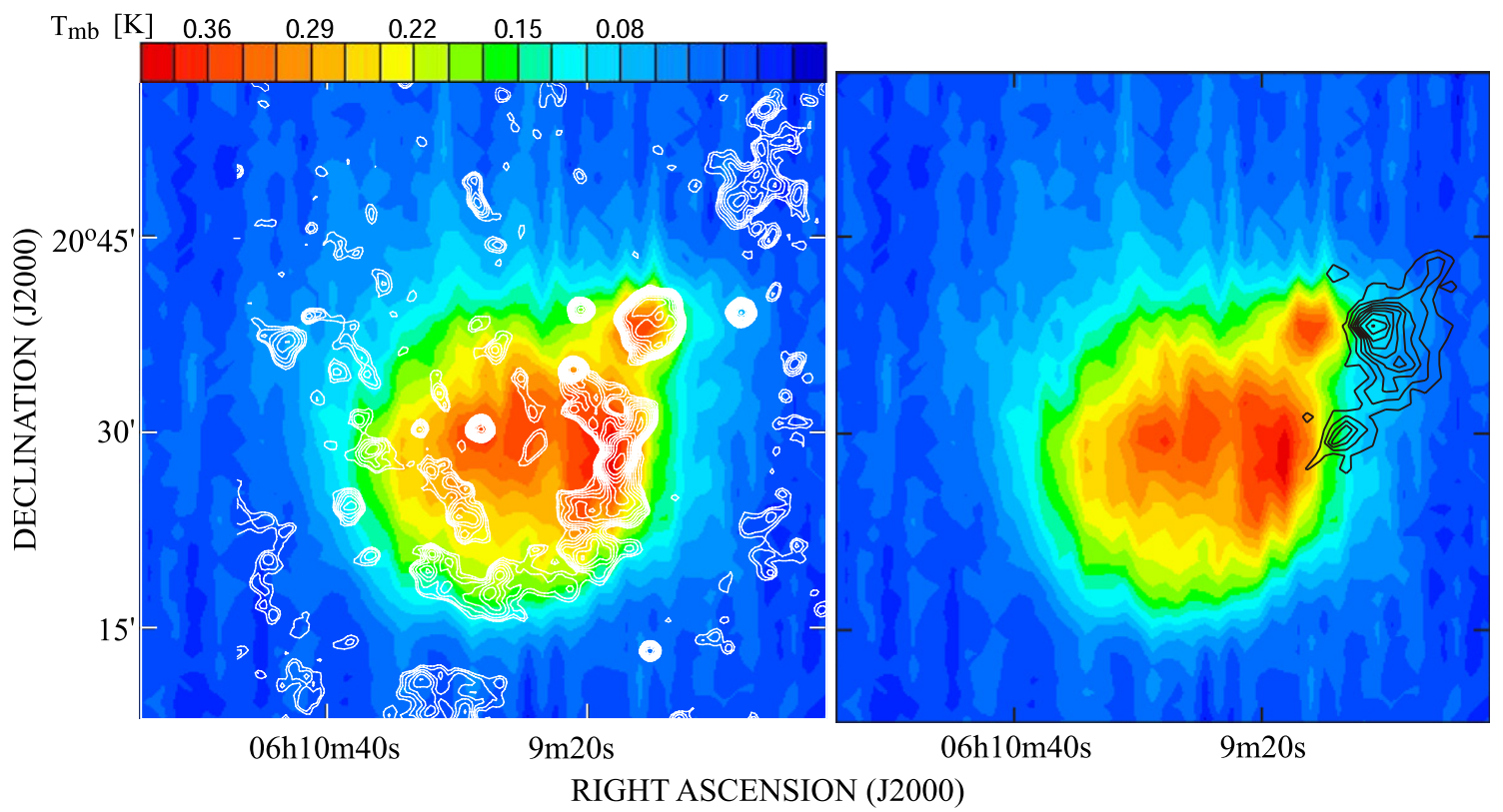

Figure 5. Radio continuum map obtained with the Yamaguchi $32 \mathrm{~m}$ telescope at $8.4 \mathrm{GHz}$ in pseudo color. The color bar is shown on top of the map. The emission originates from the ionized gas in the $\mathrm{H}$ II region. The radio continuum emission map obtained with VLA at $1.4 \mathrm{GHz}$ is shown by white contours on our pseudo-colored continuum map (left). The relative position of the $\mathrm{NH}_{3}(1,1)$ map (Figure 2), shown with black contours, can be seen in the right panel.

(A color version of this figure is available in the online journal.)

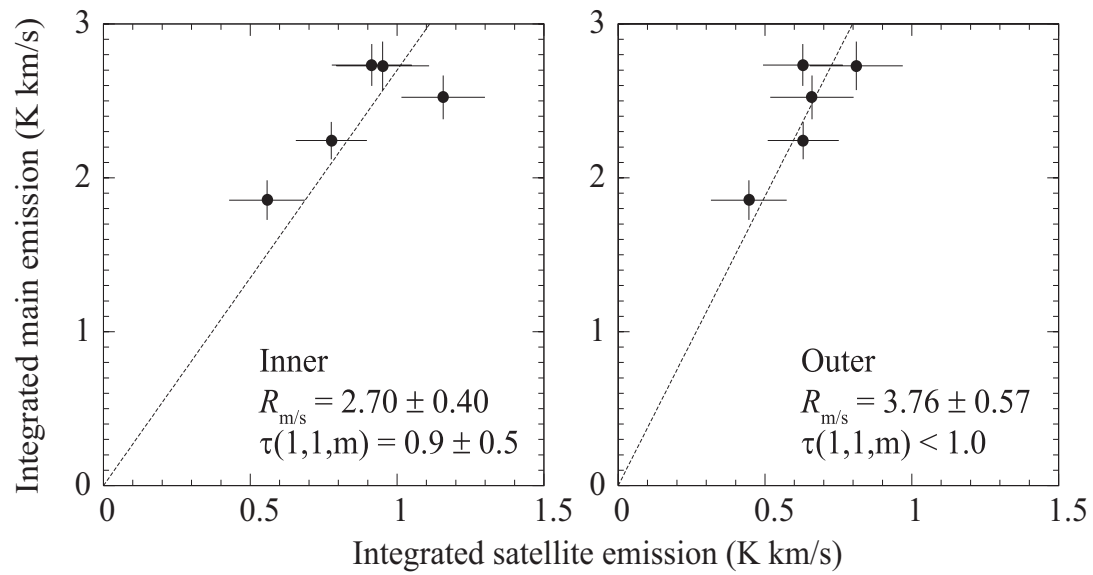

Figure 6. $\mathrm{NH}_{3}(1,1)$ correlation between the main lines and the inner and outer satellite lines (see Figure 1$) . R_{\mathrm{m} / \mathrm{s}}$ is the integrated intensity ratio of the $(1,1)$ main line to the inner and outer satellite lines.

The rotational temperature of $\mathrm{NH}_{3}, T_{\text {rot }}$, is estimated using the intensity ratio of the $(2,2)$ line to the $(1,1)$ line with the optical depth estimated through

$T_{\mathrm{rot}}=-41.5 / \ln \left(\frac{-0.282}{\tau} \times \ln \left[1-\frac{T_{\mathrm{MB}}(2,2)}{T_{\mathrm{MB}}(1,1)} \times\{1-\exp [-\tau]\}\right]\right)$,

where $\tau$ is the optical depth of the $(1,1)$ main line, and $T_{\mathrm{MB}}(2,2)$ and $T_{\mathrm{MB}}(1,1)$ are the brightness temperatures of the $(1,1)$ and $(2,2)$ main lines, respectively. We used the main beam brightness temperature as the brightness temperature. The observed ratios of $T_{\mathrm{MB}}(2,2)$ to $T_{\mathrm{MB}}(1,1)$ range between 0.2 and 0.5 at the detected line positions. Using the collisional excitation model (Walmsley \& Ungerechts 1983; Danby et al. 1988), we can derive the gas kinetic temperature, $T_{\text {kin }}$, from $T_{\text {rot }}$. We estimated it at 33 positions where both the $(1,1)$ and $(2,2)$ lines are detected over the $3 \sigma$ level, and they range between 10 and $50 \mathrm{~K}$.
The distribution of the resultant gas kinetic temperatures within the dense molecular cloud and a histogram of the kinetic temperatures are shown in Figure 7. From Figure 7 (left panel), it is evident that the warmest gas is distributed $6^{\prime}$ or $3.7 \mathrm{pc}$ from the western edge of the MHN and beyond the main ridge of the molecular cloud. This warmest gas is located at a compact H II region called S252A (Shepherd \& Churchwell 1996; Bronfman et al. 1996), which has its isothermal dust mass estimated to be $220 M_{\odot}$ (Mueller et al. 2002). The rest of the molecular gas distribution including along the MHN are relatively colder than those around S252A. The histogram shows that most parts of the molecular cloud temperature range from 10 to $30 \mathrm{~K}$.

\subsection{Mass of the Molecular Cloud}

The mass of the dense molecular cloud is critical for estimating the star formation activity in the region. We derived the local thermal equilibrium (LTE) and virial masses of the molecular cloud. The LTE mass is estimated from the line intensity with an 

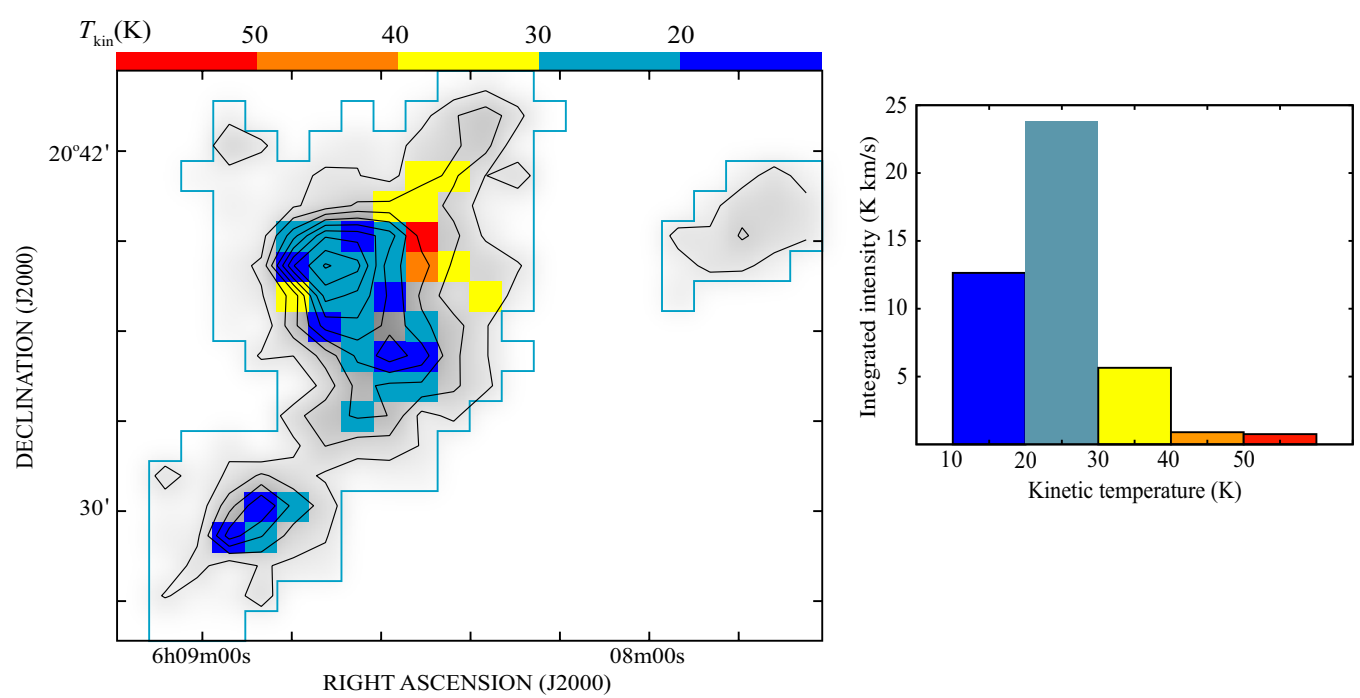

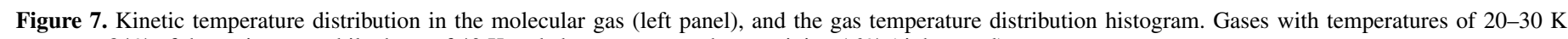
represent $84 \%$ of the entire gas, while those of $40 \mathrm{~K}$ and above represent the remaining $16 \%$ (right panel).

assumption of the LTE. Using the line intensity, optical depth, and gas kinetic temperature, we can estimate the total column density through

$$
\begin{aligned}
N\left(\mathrm{NH}_{3}\right)= & N(1,1)\left[\frac{1}{3} e^{23.4 / T_{\mathrm{rot}}}+1+\frac{5}{3} e^{-41.5 / T_{\mathrm{rot}}}+\frac{14}{3} e^{-99.6 / T_{\mathrm{rot}}}\right. \\
& \left.+\cdots+\frac{2 g_{J} g_{I} g_{K}}{3} e^{\left\{(23.4-E(J, K)) / T_{\mathrm{rot}}\right\}}\right]
\end{aligned}
$$

where $E(J, K)$ is the energy of the inversion state above the ground state in units of Kelvin, $g_{J}$ is the rotational degeneracy, $g_{I}$ is the nuclear spin degeneracy, $g_{K}$ is the $K$-degeneracy (Turner $1991)$, and $N(1,1)$ is the column density of $\mathrm{NH}_{3}(1,1)$.

We obtained the column density of $\mathrm{NH}_{3}(1,1)$ to be $1.2 \times$ $10^{15} \mathrm{~cm}^{-2}$ and the total $\mathrm{NH}_{3}$ column density, $N\left(\mathrm{NH}_{3}\right)$, is $2.9 \times$ $10^{15} \mathrm{~cm}^{-2}$.

The LTE mass is given as the integration of the column density over the cloud with the $\mathrm{NH}_{3}$ abundance $X\left(\mathrm{NH}_{3}\right)$ relative to hydrogen, $\mathrm{H}_{2}$. There have been many studies of the $\mathrm{NH}_{3}$ abundance. Ho \& Townes (1983) estimated the abundance to be $10^{-7}$ for the dense molecular core of the L183 dark core (Ungrerechts et al. 1980), and $10^{-5}$ in the hot core of the Orion KL (Genzel et al. 1982). Ion-molecule chemistry shows the abundance to be $10^{-8}$ (Prasad \& Huntress 1980). We assume $X\left(\mathrm{NH}_{3}\right)=10^{-7}$ after Ho \& Townes (1983). This is because our estimated kinetic temperature and the cloud size are close to those of L183. Using the $N\left(\mathrm{NH}_{3}\right)$ and the assumed $X\left(\mathrm{NH}_{3}\right)$, we obtained the hydrogen column density $N\left(\mathrm{H}_{2}\right)$ to be $2.9 \times$ $10^{22} \mathrm{~cm}^{-2}$. The cloud size was estimated from the geometric mean of the major and minor axes of an apparent ellipse after beam deconvolution. The size in diameter was estimated to be $2.3 \mathrm{pc}$. Our result shows a consistent hydrogen number density of $10^{5} \mathrm{~cm}^{-3}$. The resultant LTE mass is $1.8 \times 10^{3} M_{\odot}$.

The virial mass is estimated from FWHM and cloud sizes $(0.3,1.3,0.4,0.4 \mathrm{pc}$, cloud division shown in Figure 2) with the assumption of the virial equilibrium. We used the typical velocity width of the cloud as $2.68 \mathrm{~km} \mathrm{~s}^{-1}$, which is observed at the $\mathrm{NH}_{3}$ peak. We divided the cloud into four parts for the purpose of deriving the virial mass (see Figure 2, left panel), applied the uniform sphere model (MacLaren et al. 1988), and estimated the cloud virial mass, $M_{\mathrm{vir}}$, to be $1.75 \times 10^{3} M_{\odot}$.
Based on both mass estimations, we obtained the mass of the molecular cloud to be $\sim 2 \times 10^{3} M_{\odot}$.

\section{DISCUSSION}

\subsection{Star Formation in the Molecular Cloud}

Two major paradigms are proposed to explain the star formation activities around a diffuse $\mathrm{H}$ II region: spontaneous and sequential. Spontaneous star formation is a star-forming process starting from the simple gravitational collapse of a dense core in a dense molecular cloud without any external trigger. Assuming that Bonnell \& Bate's (2006) gravitational collapse and competitive accretion theory of massive star formation is applicable in this situation, the spontaneous star formation implies that a massive star is formed by the natural, early gravitational collapse of a core in the center of a stellar cluster (the collapse is not influenced by an expanding H II region nearby). Subsequently, it competitively accretes more materials than its counterpart by virtue of its location and early birth. Sequential star formation is a star-forming process that starts due to the effect of a massive star on the molecular gas, such as an expanding H II region (Elmegreen \& Lada 1977; Deharveng et al. 2005) or supernova shock. The star formation around the MHN was suggested to be a typical sequential star formation site (e.g., Carpenter et al. 1995b).

Figure 7 shows that the gas temperature on the eastern boundary of the molecular cloud is as cold as that of a typical molecular cloud or about $20 \mathrm{~K}$. If the dense cloud were formed by the shock due to the expansion of the MHN, the compressed gas should be heated up.

We can say that the shock heated gas was not found because the gas may have cooled down quickly enough to smear it out. However, we believe this is not the case. Figure 7 shows that the gas is as warm as $50 \mathrm{~K}$ at the back side of the cloud. This should not be due to the MHN. Our $8.4 \mathrm{GHz}$ map shows that the extent of the ionized gas is well traced by the optical image (see Figure 5).

The position-velocity diagram (Figure 8 ) across the cloud in $\mathrm{NH}_{3}$ shows a redshift at $\mathrm{S} 252 \mathrm{~A}$. S252A is associated with an embedded star cluster and the $\mathrm{H}_{2}(1-0) \mathrm{S} 1$ line on the $2.12 \mu \mathrm{m}$ image shows an arc structure between the compact $\mathrm{H}$ II region and the molecular cloud (Tej et al. 2006). It means that the 


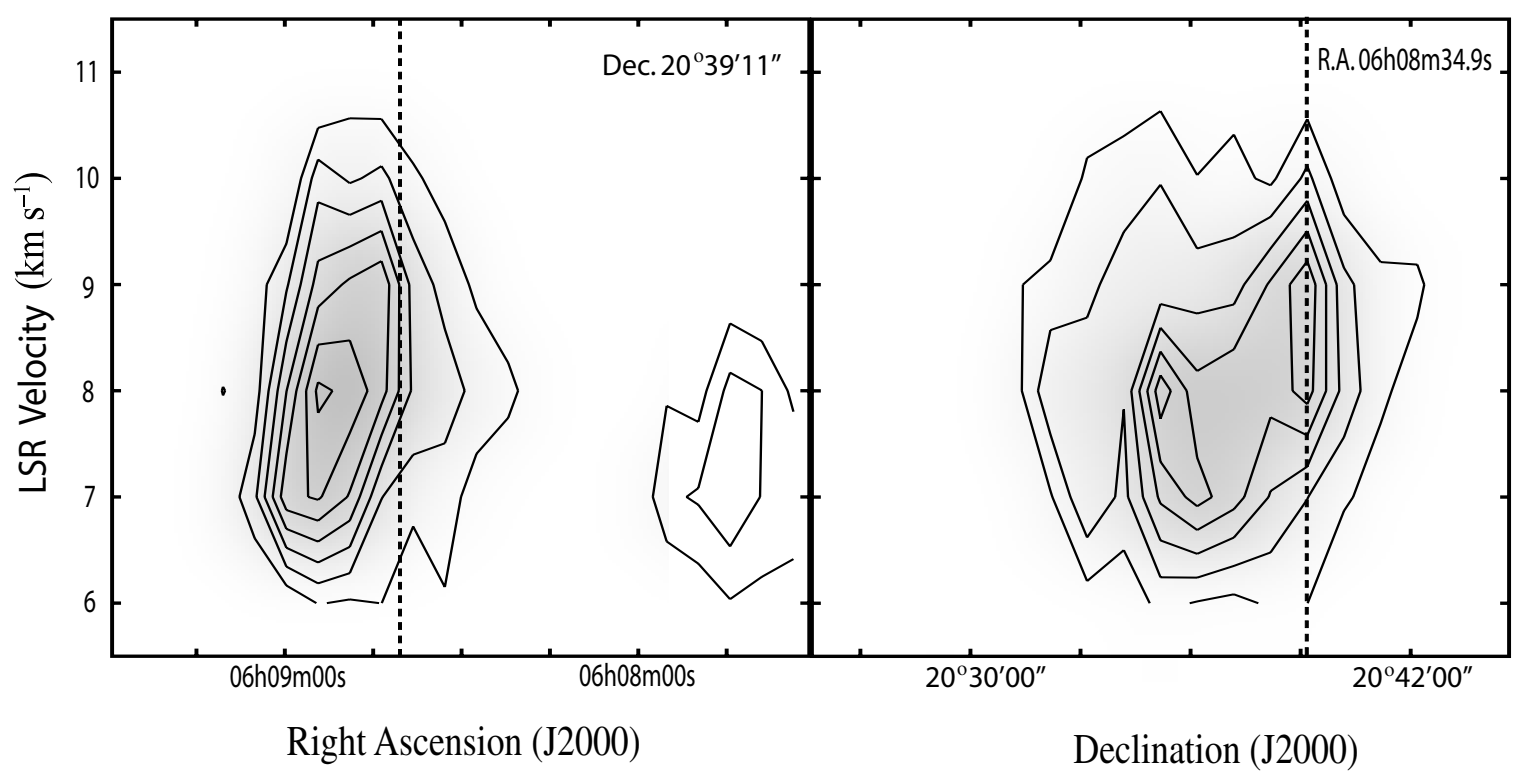

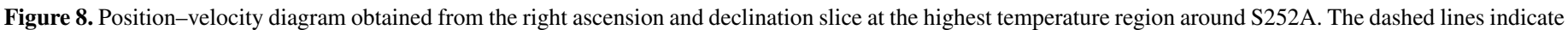
the position of the slice and the existence of contour lines on both sides of the dashed lines indicates redshift and blueshift.

compact $\mathrm{H}$ II region, $\mathrm{S} 252 \mathrm{~A}$, interacts with the dense molecular cloud. The warm molecular gas seen in our $\mathrm{NH}_{3}$ map should be a result of this interaction.

\subsection{Distribution of Young Stellar Objects}

To investigate the star formation activities in the region, we explored the distribution of YSOs. This was obtained from the point-source catalog (PSC) with the Wide-field Infrared Survey Explorer (WISE) archived at the NASA/IPAC Infrared Science Archive operated by the Jet Propulsion Laboratory (JPL).

The WISE catalog covers the whole sky in four bands: $3.4 \mu \mathrm{m}$ (Band 1), $4.6 \mu \mathrm{m}$ (Band 2), $12 \mu \mathrm{m}$ (Band 3), and $22 \mu \mathrm{m}$ (Band 4). The angular resolutions are 6.'1, 6."4, 6.'5, and 12".0 for Bands 1 to 4 , respectively. We search the WISE/2MASS sources centered at $(\alpha, \delta)_{\mathrm{J} 2000}=\left(06^{\mathrm{h}} 09^{\mathrm{m}} 06^{\mathrm{s}} 00,+20^{\circ} 33^{\prime \prime} 00^{\prime \prime} \cdot 0\right)$ within a $40^{\prime} \times 40^{\prime}$ area. We found about 5927 unclassified point sources from the catalog.

In order to classify the obtained point sources in class I and II YSOs, we employed the procedure shown in Koenig et al. (2012), which adapted the classification scheme developed by Gutermuth et al. (2008, 2009). This procedure includes the rejection of extragalactic sources, shock emission blobs, and resolved structures. Using the WISE band 4 or $22 \mu \mathrm{m}$ and the positionally associated Two Micron All Sky Survey (2MASS) $K_{s}$-band data, we retrieve some of the reddened class II objects from transition disks, which are disks that have a photospheric color between $3.4 \mu \mathrm{m}$ and $12 \mu \mathrm{m}$ but an excess at $22 \mu \mathrm{m}$, following the procedure in the Appendix of Koenig et al. (2012). While Gutermuth and collaborators, as well as Koenig and collaborators, created extinction maps to deredden the sources, we used the extinction value from the extinction map based on the 2MASS PSC created by Dobashi (2011).

The classification procedure gave rise to 175 class I YSOs, 268 class II YSOs, and 15 transition disk sources. Before delving into our analyses and classification of the YSOs, we want to note that we assumed the YSOs to be of low and intermediate masses. There may be a few massive protostars among them, but their number is insignificant and does not alter the overall judgment based on the YSO distribution. Our judgment is supported by the 2MASS color-magnitude diagram of WISE selected YSOs (class I, class II, and transition disk). The total number of YSOs with 2MASS $J$ - and $H$-band data is 253 . You can see that 155 (about $60 \%$ ) WISE selected YSOs have a mass of $M>2 M_{\odot}$. We adopt the 3 Myr evolutionary track by Siess et al. (2000). Stars with $\mathrm{M}>3 M_{\odot}$ have already entered the main sequence at $3 \mathrm{Myr}$ (see Figure 9) and thus should be visible in the optical if they are massive enough to have formed compact $\mathrm{H}$ II regions. Minier et al. (2005) reported two methanol masers around the region associated with $M S X$ sources. We have also checked the $M S X$ catalog of the region and detected some YSOs associated with bright $\mathrm{H}$ II regions and intermediate-mass stars.

The distribution of all the obtained YSOs is shown in Figure 10. We can find both class I and II YSOs throughout the observed region, although some are concentrated around the compact H II region S252A, S252C (Felli et al. 1977), the bright $\mathrm{H} \alpha$ condensation east of $\mathrm{S} 252 \mathrm{~A}$, which is associated with the NGC 2175H134 O6V star (Haikala 1994), and the ultracompact $\mathrm{H}$ II region positionally corresponding to the small $\mathrm{NH}_{3}$ located west of S252A, which positionally corresponds to IRAS $06047+2040$.

These YSO concentrations should be due to the triggered star formation by these three H II regions. However, other YSOs are distributed rather uniformly. If most stars in this region were formed by the expansion of the MHN, these YSOs would show shell-like distribution after the propagation of star formation activity. A lot of YSOs beyond the dense molecular cloud cannot be made by the assumed expanding shell, because the shell should be located near the molecular cloud. Moreover, we cannot find any systematic age difference of YSOs over the region. All of these results reject that these YSOs are made by an expanding shell associated with the MHN. They should have been formed randomly. This means that most of the YSOs in this region were made spontaneously.

\subsection{Evidence of Small-scale Triggered Star Formation}

We found that neither molecular gas nor YSOs in the MHN region show any systematic evidence of large-scale triggered star formation due to the MHN. This means that the sequential 


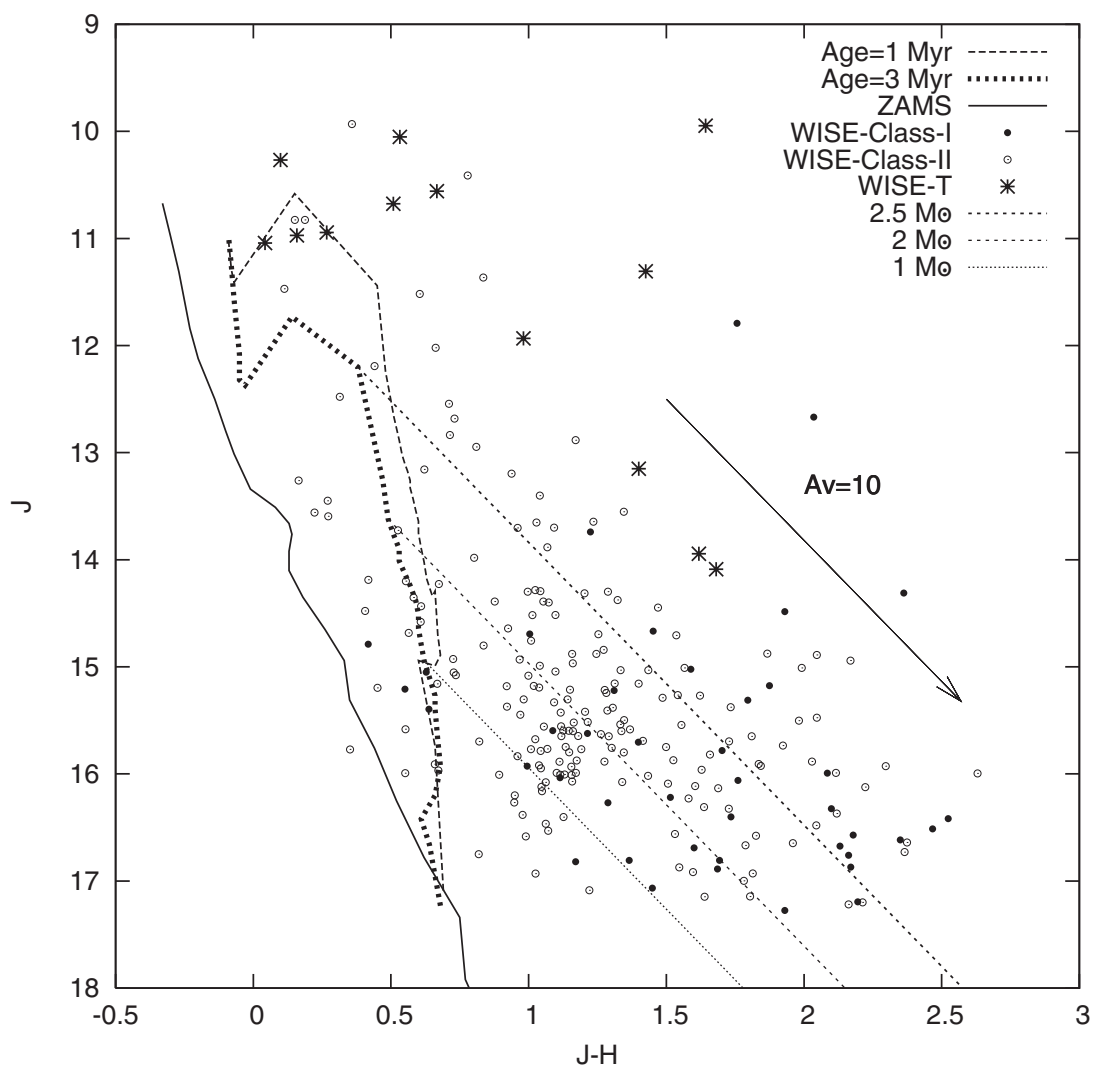

Figure 9. 2MASS color-magnitude diagram of the WISE-selected YSOs in the MHN. The ZAMS line is the zero age main-sequence line, while the WISE-T are the WISE-selected transition disk sources. Most of the low- and intermediate-mass stars lie above the $3 \mathrm{Myr}$ line.

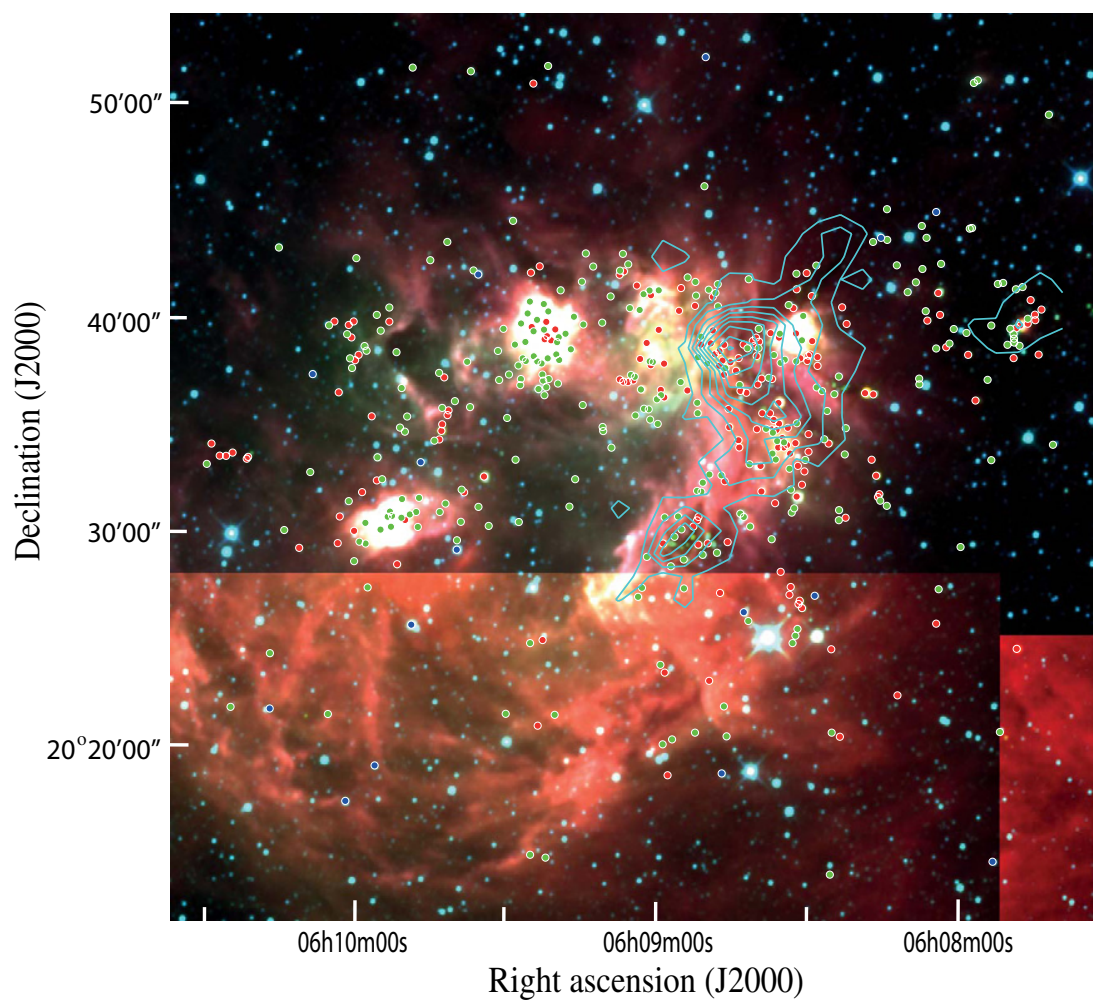

Figure 10. Distribution of the WISE/2MASS class I (red circles), II (green circles) YSOs, and the transition disk sources (blue circles) with $\mathrm{NH}_{3}(1,1)$ contour map overlaid on the WISE image of the region.

(A color version of this figure is available in the online journal.) 
star formation does not take place there. However, we cannot discard all triggered star formation activity in this region.

We found temperature enhancement in the molecular gas associated with the compact $\mathrm{H}$ II region S252A (Figure 7). From the WISE archive data we found that there are YSO concentrations in the compact $\mathrm{HII}$ regions (Figure 10). Tej et al. (2006) found a bow shock associated with S252A in the shock molecular hydrogen line. The shock is located toward the dense molecular cloud. Bonatto \& Bica (2011) suggested that the embedded star cluster in S252A was made in a coeval star formation event with $\pm 5 \mathrm{Myr}$ and extended for about $10 \mathrm{Myr}$, and that the cluster was made after the single star formation event. Our WISE/2MASS results support this because we did not find any hierarchal distribution of the class II and class I YSOs, which would have been evidence of their formation occurring due to two different events separated by a time lag.

All of these results show that there is some effect of an expanding $\mathrm{H}$ II region on nearby molecular gas. However, it is very limited both in spatial and mass scales. The warmest part of the dense molecular cloud is 0.9 pc apart from S252A. The molecular gas temperature map (Figure 7) shows that the warmest gas size is smaller than our beam size and is distributed $3.7 \mathrm{pc}$ west of the MHN. Therefore, we conclude that the expanding $\mathrm{H}$ II region of S252A affects an area as large as 0.9 pc. We found YSO concentration to two compact H II regions. However, all these YSOs are less massive ones and we cannot find any massive YSOs associated with these compact $\mathrm{H}$ il regions.

\subsection{OB Star Formation Scenario in the MHN}

If the effect of MHN on the surrounding interstellar matter is limited, how should we explain the association of the dense molecular gas and MHN? Our radio continuum mapping shows that the $\mathrm{H}$ II region and the dense molecular cloud are physically in contact; this is not an apparent association by chance on the same line of sight nor is it extinction due to the dense molecular cloud.

We propose that the dense molecular cloud is not formed by the expansion of the MHN but is present before the MHN expansion. At that time there were several dense gas cores in this region. At first, the most massive core collapsed and became the O6.5V star HD 42088, which is the exciting star of the whole MHN (Haikala 1994). Although the expansion of the $\mathrm{H}$ II region affected the interstellar matter around it, its effect was limited. About 1 Myr later, some cores collapsed spontaneously and formed some compact $\mathrm{H}$ II regions within this region. S252A is among the youngest of them. During this short timescale, a lot of YSOs simultaneously were formed from the dense gas cloud.

This scenario can also explain why the dense molecular cloud is located on the northwestern edge of the MHN. Figure 3 shows that the MHN edge is even less associated even with the ${ }^{13} \mathrm{CO}$ map morphologically. We observed no significant emission in the $\mathrm{NH}_{3}$ line at a few positions of these ${ }^{13} \mathrm{CO}$ peaks, for example at $(\alpha, \delta)_{\mathrm{J} 2000}=\left(06^{\mathrm{h}} 09^{\mathrm{m}} 26^{\mathrm{s}} .51,+20^{\circ} 39^{\prime} 10^{\prime \prime} \cdot 70\right)$.

\section{SUMMARY}

We presented the results of our $\mathrm{NH}_{3}$ line and continuum (ionized gas) emission observations toward the MHN. From our observations we have obtained the physical parameters and temperature distribution of the dense molecular gas distributed northwest to southeast of the MHN. Evidently, the region around the S252A compact $\mathrm{H}$ II region has higher temperatures compared to the rest of the molecular gas, including the interface between the dense molecular cloud and the MHN. This is due to the heating effect of the radiation wind from the exciting star of the S252A compact $\mathrm{H}$ II region and does not seem to have anything to do with the extended $\mathrm{H}$ II region of the MHN. We have also explored the star formation scenario in the region and argue that the OB stars in the region form spontaneously rather than sequentially due to the effect of the expanding $\mathrm{H}$ II region.

The molecular gas region traced in our $\mathrm{NH}_{3}$ observations should have originally formed and spontaneously collapsed leading to the formation of stars rather than star formation being triggered by the sweeping together of materials.

The overall distribution of low- and intermediate-mass stars obtained with WISE/2MASS (see Koenig et al. 2012) in the region supports the spontaneous star formation scenario. However, the localized triggered formation of the low- and intermediate-mass stars around the $\mathrm{S} 252 \mathrm{~A}$ compact $\mathrm{H}$ II region and two other HiI regions are favored by our WISE/2MASS results. Though triggered star formation may be happening in limited portions of the entire region, we do not think that it is responsible for the formation of the massive $\mathrm{OB}$ stars in the Gem OB1.

We thank the anonymous referee for the constructive and valuable comments that helped improve our manuscript. We wish to acknowledge the support of the staff members of the Astrophysics group of Kagoshima University. Our heartfelt appreciation also goes to the NICT $34 \mathrm{~m}$ Kashima and $32 \mathrm{~m}$ Yamaguchi radio telescope operation teams for their assistance and kind support. J.O.C. has been financially supported by the Ministry of Education, Culture, Sports, Science, and Technology (MEXT) of Japan. T.H. is supported in part by the MEXT Grant-in-Aid for Specially Promoted Research (Tokubetsusuishin) No. 20001003. The Wide-field Infrared Survey Explorer (WISE) is a joint project of the University of California, Los Angeles and the Jet Propulsion Laboratory (JPL), California Institute of Technology (Caltech) and funded by the National Aeronautics and Space Administration (NASA). The Two Micron All Sky Survey (2MASS) is a joint project of the University of Massachusetts and the Infrared Processing and Analysis Center (IPAC)/Caltech, funded by NASA and the National Science Foundation.

\section{REFERENCES}

Bonatto, C., \& Bica, E. 2011, MNRAS, 414, 3796

Bonnell, I. A., \& Bate, M. R. 2006, MNRAS, 370, 488

Bronfman, L., Nyman, L.-A., \& May, J. 1996, A\&AS, 115, 81

Carpenter, J. M., Snell, R. L., \& Schloerb, F. P. 1995a, ApJ, 445, 246 Carpenter, J. M., Snell, R. L., \& Schloerb, F. P. 1995b, ApJ, 450, 201 Danby, G., Flower, D. R., Valiron, P., et al. 1988, MNRAS, 235, 229 Deharveng, L., Zavagno, A., \& Caplan, J. 2005, A\&A, 433, 565

Deharveng, L., Zavagno, A., Schuller, F., et al. 2009, A\&A, 496, 177 Dobashi, K. 2011, PASJ, 63, S1

Efremov, Y. N. 1995, AJ, 110, 2757

Elmegreen, B. G., \& Lada, C. J. 1977, ApJ, 214, 725

Felli, M., Habing, H. J., \& Israel, F. P. 1977, A\&A, 59, 43

Fountain, W. F., Gary, G. A., \& ODell, C. R. 1983, ApJ, 273, 639

Genzel, R., Ho, P. T. P., Bieging, J., et al. 1982, ApJL, 259, 103

Gutermuth, R. A., Megeath, S. T., Myers, P. C., et al. 2009, ApJS, 184, 18 Gutermuth, R. A., Myers, P. C., Megeath, S. T., et al. 2008, ApJ, 674, 336 Haikala, L. K. 1994, A\&AS, 108, 643

Ho, P. T. P., \& Townes, C. H. 1983, ARA\&A, 21, 239

Koenig, X. P., Allen, L. E., Gutermuth, R. A., et al. 2008, ApJ, 688, 1142 Koenig, X. P., Leisawitz, D. T., Benford, D. J., et al. 2012, ApJ, 744, 130 Kronberger, M., Teutsch, P., Alessi, B., et al. 2006, A\&A, 447, 921

Kutner, M. L., \& Ulich, B. L. 1981, ApJ, 250, 341 
MacLaren, I., Richardson, K. M., \& Wolfendale, A. W. 1988, ApJ, 333, 821 Minier, V., Burton, M. G., Hill, T., et al. 2005, A\&A, 429, 945

Morgan, W. W., Whitford, A. E., \& Code, A. D. 1953, ApJ, 118, 318

Mueller, K. E., Shirley, Y. L., Evans, N. J., II, et al. 2002, ApJS, 143, 469

Nakajima, T., Kaiden, M., Korogi, J., et al. 2007, PASJ, 59, 1005

Prasad, S. S., \& Huntress, W. T., Jr. 1980, ApJS, 43, 1

Reid, K. M., Zheng, X. W., Brunthaler, A., et al. 2009, ApJ, 700, 137

Sharpless, S. 1959, ApJS, 4, 257

Shepherd, D. S., \& Churchwell, E. 1996, ApJ, 472, 225
Siess, L., Dufour, E., \& Forestini, M. 2000, A\&A, 358, 593

Sofue, Y., \& Reich, W. 1979, A\&AS, 38, 251

Tej, A., Ojha, D. K., Ghosh, S. K., et al. 2006, A\&A, 452, 203

Thompson, M. A., Urquhart, J. S., Moore, T. J. T., et al. 2012, MNRAS, 421, 408

Turner, B. E. 1991, ApJS, 76, 617

Ungerechts, H., Walmsley, C. M., \& Winnewisser, G. 1980, A\&A, 88, 259

Walmsley, C. M., \& Ungerechts, H. 1983, A\&A, 122, 164

Wu, Y. W., Xu, Y., Pandian, J. D., et al. 2010, ApJ, 720, 392 